\title{
Medially-invasive cholesteatoma
}

\author{
Petar Rouev \\ ENT Dept., Trakia Hospital Stara Zagora, Bulgaria
}

\begin{abstract}
Cholesteatoma is life-threatening disease due to its intracranial complications. Some of cholesteatoma cases we define as aggressive cholesteatoma. In this category we include children cholesteatoma, medially-invasive cholesteatoma and petrous apex cholesteatoma which does not originate in the petrous apex. Another special form of cholesteatoma is petrous bone cholesteatoma. Retrospective study of 176 cholesteatoma operations (38.85\%) from 453 consecutive middle ear surgeries for 4 years and 4 months in Trakia Park Hospital Stara Zagora, Bulgaria were rewieved. The same surgeon performs 106 Primary Cholesteatoma Surgery, 49 Revision Surgery and 21 Second-Look Surgery. In this 176 cholesteatoma cases the cholesteatoma matrix involve the otic capsule, petrous apex or skull base in 5 patients, who are defined as medially-invasive cholesteatoma $(2.84 \%)$. In this medially-invasive cholesteatoma patients surgical resection with canal wall down operation remains the best treatment option.

Key words: cholesteatoma, aggressive cholesteatoma, mediallyinvasive cholesteatoma
\end{abstract}

\section{Introduction}

Cholesteatoma is a well-demarcated noncancerous cystic lesion derived from an abnormal growth of keratinizing squamous epithelium in the temporal bone, which is commonly characterized as wrong "skin in the wrong place" [1]. Cholesteatoma results from the enzymatic activity of the cholesteatoma matrix. Its abnormal growth is locally invasive and capable of causing the destruction of structures in the middle ear. The cortical temporal bone and otic capsule unlike the cellular system of the mastoid process are difficult to resorb, but in rare cases, the destruction may be in the medial direction deep into the temporal bone. When cholesteatoma matrix involve the otic capsule, petrous apex or skull base we call it medially-invasive cholesteatoma [2]. The surgical strategy is based of cholesteatoma location seen at computed tomography and preoperative audiovestibular status preserving the function of the facial nerve [3]. Apical and supralabyrinthine lesions can be treated by a middle fossa approach, infralabyrinthine and translabyrinthine locations can be exposed through a subtotal petrosectomy or a transotic route, and the retrolabyrinthine lesion can be approached via retrolabyrinthine route. Some of cholesteatoma cases we define as aggressive cholesteatoma. In this category we include children cholesteatoma, medially-invasive cholesteatoma and petrous apex cholesteatoma which does not originate in the petrous apex. Another special form of cholesteatoma is petrous bone cholesteatoma $[4,5]$. Subtotal petrosectomy is defined as complete exenteration 
of all pneumatic cell tracts of the temporal bone with or without removal of the otic capsule. $[6,7]$.

\section{Material and methods}

Retrospective study of 176 cholesteatoma operations $(38.85 \%)$ from 453 consecutive middle ear surgeries for 4 years and 4 months in Trakia Park Hospital Stara Zagora, Bulgaria. The same surgeon performs 106 Primary Cholesteatoma Surgery (60.23\%, $\mathrm{n}=176), 49$ Revision Surgery (27.84\%, $\mathrm{n}=176$; previous operations in another hospital) and 21 Second-Look Surgery $(11.73 \%, n=176)$. Sex and side distribution is: Male 88, Female 67; Left ear 78, Right ear 77. The Bellucci Classificationdistribution is: Gr. 1/5\%; Gr. 2/21\%; Gr. $3 / 68 \%$; Gr. $4 / 6 \%$. 155 patients aged 4 to 76 years (mean age 35.8 years; SD 18.0 years) were surgically treatment and included for follow-up from 24 to 60 months (mean 31.34 months; SD 13.10) between 01.06.2014 and 30.09.2018. 5 patients from them are defined as medially-invasive cholesteatoma. This was manly young people (average age 26.8; SD 10.06), 4 male and 1 female, 3 right 2 left side. All patients have Bellucci classification $\mathrm{Gr}$ 3/4. The follow-up included surveillance with clinical examination and audiometry on 3, 6, 12 months after surgery plus DWI-MRI on 18 months after surgery; then once a year. MRI has been found to be of high sensitivity and specificity in diagnosing residual or reccurent cholesteatoma, especially diffusion-weighted (DW) imaging with turbo-spin echo (TSE) [8]. Second-look surgery is usually performed on 18 months after surgery, such as the indication for that must be placed of the end of previous operation

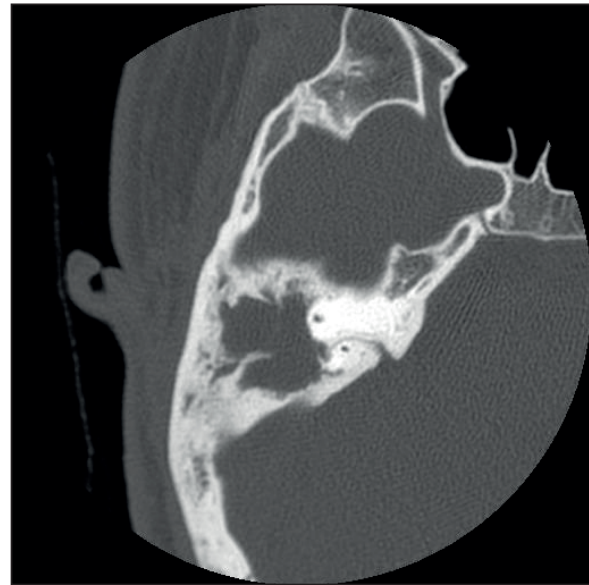

Fig. 1.1. Axial CT Scan from mediallyinvasive congenital cholesteatoma on the right side.

\section{Results}

In all patients surgical resection remains the best treatment option for this medially-invasive cholesteatoma. Our surgical principles include: (I) Need to provide bony explosure aroud disease; (II) Special attention to the facial nerve, dura mater or carotid artery; (III) When CSF leak is a concern, a subtotal petrosectomy with closure of the ear is often necessary. The patients were assessed for postoperative audiologic and facial functions and for recurrence of cholesteatoma. One has persistent otorrhea (Case 1 / Fig. 1.1, Fig. 1.2); one has dizziness (Case 2 / Fig. 2.1, Fig. 2.2, Fig. 2.3) and one has intermittent facial paresis (Case 3 / Fig. 3.1, Fig. 3.2, Fig. 3.3, Fig. 3.4, Fig. 3.5)

\section{Discussions}

Cholesteatoma is life-threatening disease due to its intracranial complications. In the past the surgeon used open techniques to treat the infectious disease and to safe the life. In the XX century the surgeon used closed techniques with canal reconstruction to preserve the hearing. Nowadays we used obliterations technique to eradicate the disease and to prevent the recurrence. Mastoid cavity obliteration could be done after certain removal of all epithelium from middle ear space. Following the matrix of cholesteatoma including complete cholesteatoma removal from anterior to posterior with preservation of malleus handle and $\mathrm{m}$. tensor tympani tendon if it is possible. The drill should not touch the epithelium and should avoid spreading of cholesteatoma particles. Before reconstruction inspection with $70^{\circ}$ endoscopes is needed to



Fig. 1.2. Axial CT Scan from mediallyinvasive congenital cholesteatoma on the right side. 


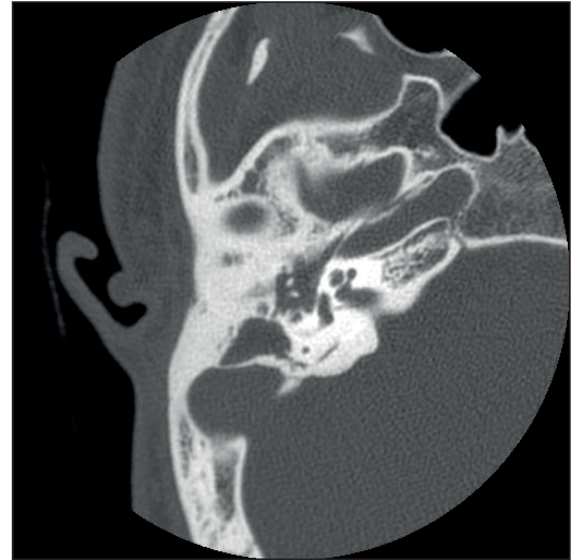

Fig. 2.1. Axial CT Scan from mediallyinvasive cholesteatoma to the posterior semicircular canal on the right side.

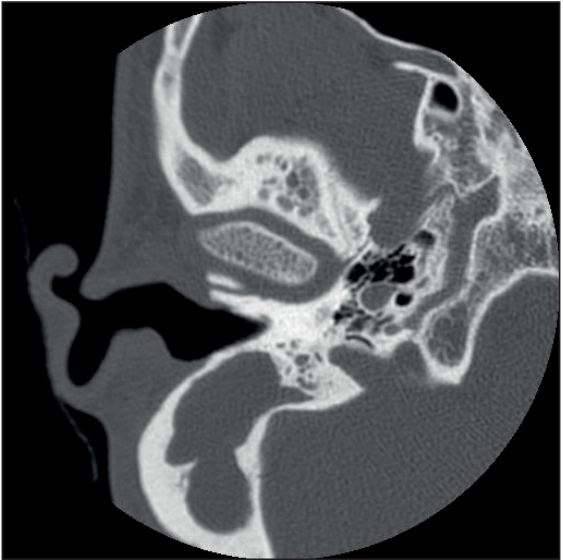

Fig. 2.2. Axial CT Scan from mediallyinvasive cholesteatoma to the posterior semicircular canal on the right side.

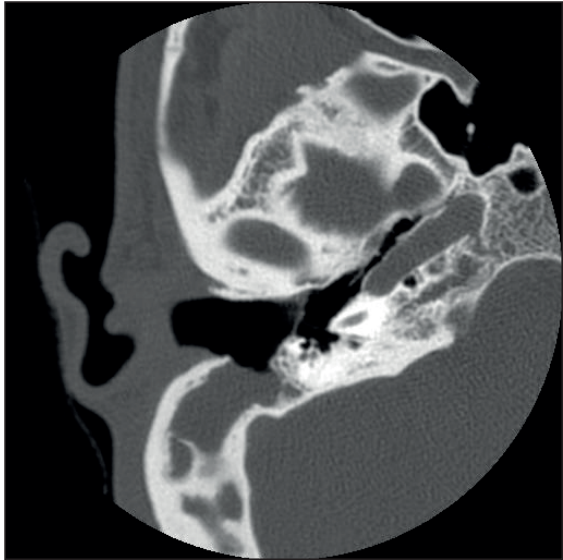

Fig. 2.3. Axial CT Scan from mediallyinvasive cholesteatoma to the posterior semicircular canal on the right side.

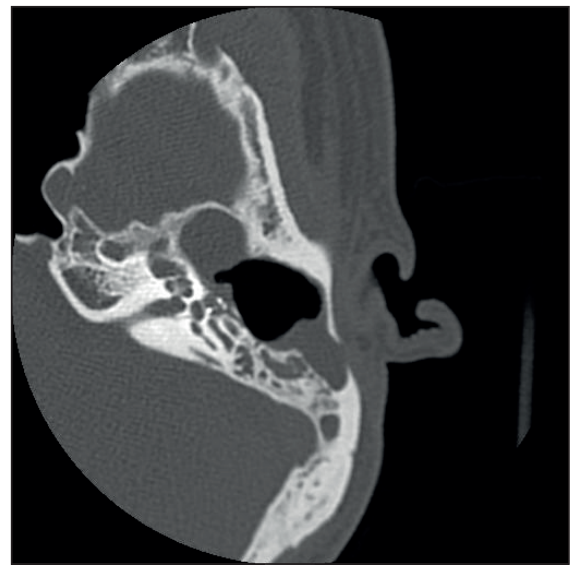

Fig. 3.1. Petrous apex cholesteatoma on the left side.

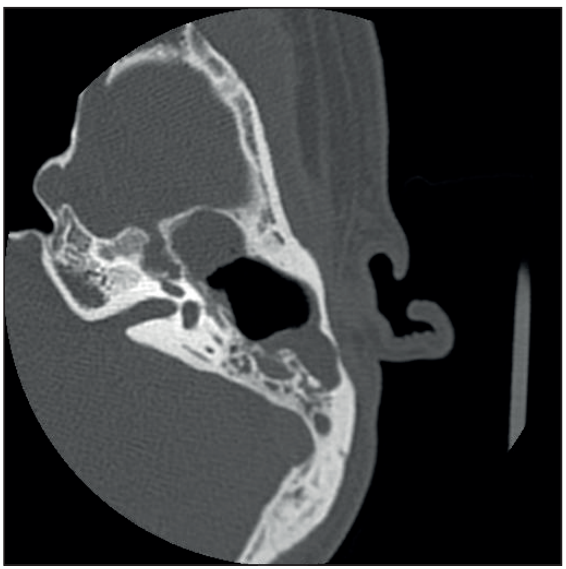

Fig. 3.2. Petrous apex cholesteatoma on the left side.

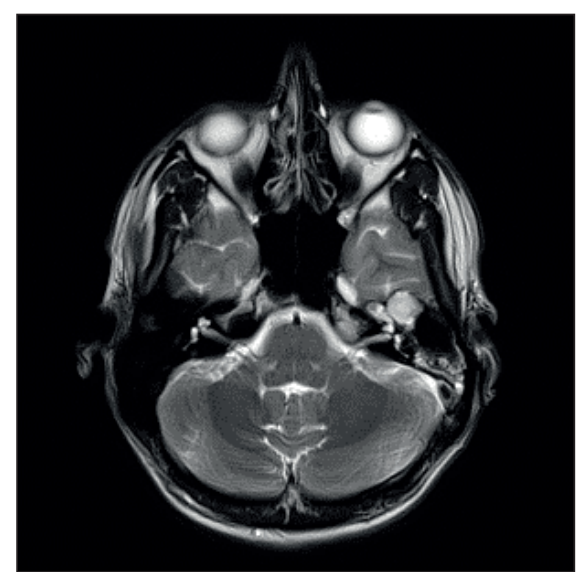

Fig. 3.3. DWI-MRI (diffusion-weighted imaging with turbo-spin echo) 18 months after surgery display the residual cholesteatoma in petrous apex area on the left side.

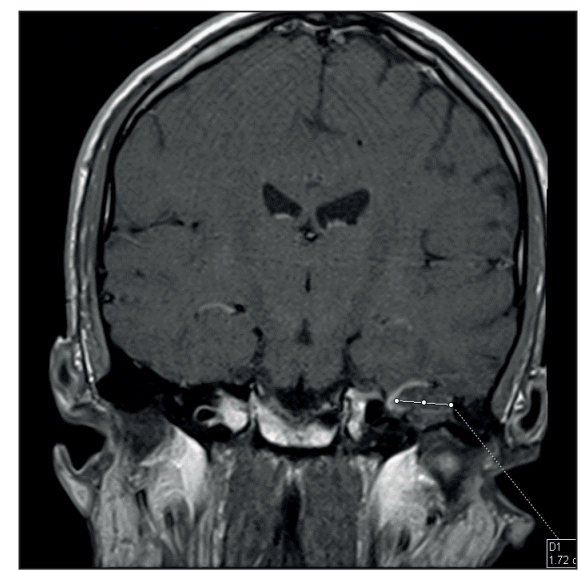

Fig. 3.4. Horizontal diameter on the lesion (D1=1.72 cm).

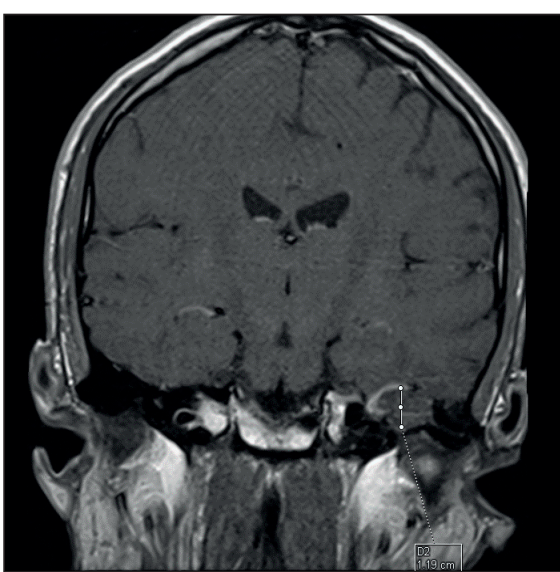

Fig. 3.5. Vertical diameter on the lesion (D2=1.19 cm). 
be sure, that all epitelium is removed. This obliteration procedure is designed to prevent development of postoperative retraction pockets by obliterating the mastoid cavity using bone pate or synthetic bone, covered with cartilage or connective tissue and resulting isolation the tympanum from the attic and mastoid.

The surgeon's experience is essential to the success of cholesteatoma surgery. The so-called "drill guy" is the professional, who builds in the temporal bone laboratory, and who is in fact a key person in reducing the cases with recurrence of cholesteatoma. In cases of medially-invasive cholesteatoma otologic surgeon (Middle ear; Ossiculoplasties; Mastoidectomy) goes to temporal bone surgeon (Subtotal Petrosectomy; Petrous Apex Surgery) to get to lateral skull base surgeon or neurosurgeon (Subtotal Petrosectomy; Middle Fossa Surgery; Transotic/Translabyrinthine route surgery; Infratemporal Fossa Approach Type A, B, C a modo Fish etc.).

\section{Conclusion}

Our experience shows that medially invasive choleateatoma are observed exclusively in patients with prolonged and resistant to treatment otorrhea.
The CT scans of these patient shows well-developed mastoid pneumatization. The medially-invasive cholesteatoma demonstrates an aggressive, endophytic growth pattern, invading into the otic capsule or through the perilabyrinthine air cells to the petrous apex. Surgical resection with canal wall down surgery remains the best treatment option for medially-invasive cholesteatoma. In some cases may be necessary transcochlear or skull-base approaches to eradicate the disease and the cholesteatoma matrix may be adherent to facial nerve and membranous labyrinth. We must be having special attention when engaging the carotid artery or jugular bulb, such as in this cases infratemporal fossa approach is preferable

\section{Compliance with ethical standards}

Disclosure of potential conflicts of Interest: The author have no conflict of interest to declare. Financial Disclosure: The author declared that this study has received no financial support. Informed Content: This articles does not contain patient data. This study was presented as ePoster at "HNO-Online-Kongress 2021: 92. \& 91. Jahresversammlung der DGHNO-KHC”, 12-16. May 2021.

\section{References}

1. Robinson J. M., Cholesteatoma: skin in the wrong place. Journal of the Royal Society of Medicine, 1997; 90(2): 93-96.

2. Geoffrey Casazza, Matthew L Carlson, Clough Shelton, Richard K Gurgel. The Medially-Invasive Cholesteatoma: An Aggressive Subtype of a Common Pathology. Ann Otol Rhinol Laryngol. 2021; 130(1): 38-46 (doi: 10.1177/0003489420937731. Epub 2020 Jun 30).

3. Grayeli AB, Mosnier I, El Garem H, Bouccara D, Sterkers O. Extensive intratemporal cholesteatoma: surgical strategy. Am J Otol. 2000 Nov; 21(6): 774-81.

4. Mario Sanna 1, Yash Pandya, Fernando Mancini, Giuliano Sequino, Enrico Piccirillo. Petrous bone cholesteatoma: classification, management and review of the literature. Audiol Neurootol. 2011; 16(2): 124-36. (doi: 10.1159/000315900. Epub 2010 Jul 29).

5. Pascal Senn, Rudolf Haeusler, Eugène Panosetti, Marco Caversaccio. Petrous bone cholesteatoma removal with hearing preservation. Otol Neurotol. 2011 Feb; 32(2): 236-41 (doi: 10.1097/MAO.0b013e3182001ef4).

6. Giulia D’Angelo, Giulia Donati, Andrea Bacciu, Maurizio Guida, Maurizio FalcioniSubtotal petrosectomy and cochlear implantation. ACTA OTORHINOLARYNGOLOGICA ITALICA 2020; 40: 450-456; doi: 10.14639/0392-100X-N0931.

7. Free RH, Falcioni M, Di Trapani G, et al. The role of subtotal petrosectomy in cochlear implant surgery - a report of 32 cases and review on indications. Otol Neurotol 2013; 34: 1033-40. https: //doi.org/10.1097/MAO.0b013e318289841b

8. Peter M. M. C. Li, Eleni Linos, Richard K. Gurgel, Nancy J. Fischbein, Nikolas H. Blevins. Evaluating the utility of non-echo-planar diffusion-weighted imaging in the preoperative evaluation of cholesteatoma: A meta-analysis. Laryngoscope 2013; 123(5): 1247-50 (Epub 2012 Sep 28) 